\title{
DETERMINATION OF REFERENCE FUNCTION TO KNEE PROSTHESIS RATING
}

\section{Gábor Katona $^{1}$, Béla M. Csizmadia ${ }^{1}$, Kristóf Andrónyi ${ }^{2}$}

${ }^{1}$ Szent István University, Faculty of Mechanical Engineering, Institute of Mechanics and Machinery

${ }^{2}$ Uzsoki Hospital, Department of orthopedic and traumatology

katona.gabon@gek.szie.bu

\begin{abstract}
The knee joint is one of the most complicated joints in the human body. Fundamental knowledge about kinematics is essentially important in order to design knee prostheses which are functionally similar to the knee joint. In addition, the prosthesis should carry out similar kinematics, since only the geometrical resemblance is insufficient. As an initial step, an experimental apparatus, a test protocol and an evaluation method were developed which allows the investigation of the human knee joint kinematics. Based on the results of multiple experiments, a reference function was determined, which provides guidance on how the flexionrotation function in the human knee joint can be described. The function also provides reference about the kinematics which should be defined in the design of prostheses, and to what extent does an arbitrary knee prosthesis follows the required kinematics. The definition of knee rotation is reported in this article.
\end{abstract}

Keywords: biomechanics, knee joint, rotation, flexion, kinematics, reference function

\section{Introduction}

The goal of our research is to determine a kinematical and kinetical model of knee joint, which intends to help in the development of better prostheses. The movement of the knee joint can be investigated locally (sliding-rolling, etc.) ${ }^{1}$ and globally (rotation, abduction, adduction, etc). ${ }^{2}$ The rotation has the most significant role in the knee joint motion from the above-mentioned knee movements. Our current aim is to describe the rotation of the human knee joint as a function of flexion. The reason for this is twofold. On the one hand, the knee joint kinematics can be characterized by this result, and on the other hand, it can be used to qualify and design knee prostheses. A suitable prosthesis has to be similar to a real knee joint regarding the carried out movements and not the geometry.

Kinematic analyzes presented by different authors show large deviation ${ }^{3-8}$ (Figure 1). A mention must be made that several flexion-rotation functions of other authors - in accordance with reality - does not start from the origin. However, the starting point of the measured curves is transformed to the origin by most of the authors. Nevertheless, most of them do not indicate how this transformation is carried out. This problem will be further discussed in the article. 


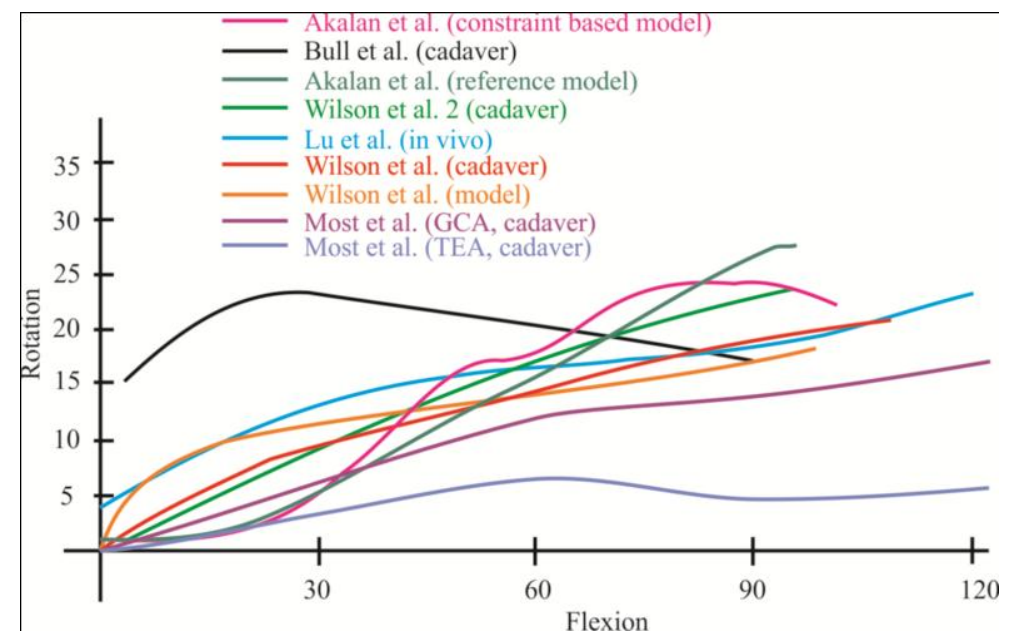

Figure 1. The results of different authors

Additional reasons for the differences between the curves are the followings: biological differences between the people, the applied experimental apparatus, the method of measurement and evaluation, different types of motion and at last the measurement error. The separation of these reasons is an important task in order to draw general conclusions. The diversity of apparatuses and the evaluation methods have significant role in the differences between the results. The upcoming errors can be significantly reduced by using simple apparatus for the experiments. For this reason, a novel, relatively simple experimental apparatus was developed by the Research Team. This apparatus helps the error analysis, the comparison of the measurements and ensures the unconstrained movement of the knee joint. One of the aims was to obtain precise and reproducible kinematical data possible about the knee joint motion.

General aim of the experimental apparatus design and construction was to carry out experiments on cadaver knee joints, in order to provide kinematic data to the knee prosthesis design. The apparatus was later further modified in a way that even prostheses can be clamped into the same apparatus, thus experiments under the same conditions can be carried out in order to compare the results of the measurements with the physiological joint kinematics. The usability of the cadaver knee joint as experimental model have only been studied by the default installation of the experimental apparatus in case of one motion type. This motion is the flexion of the tibia with a horizontally fixed femur.

The experimental apparatus is multifunctional, which is suitable for the determination of the cadaver knee kinematics and kinetics (Figure 2). It can be used in MR, in case of different type of loads and it is also suitable for testing knee prostheses. In the followings, only those parts of the apparatus will be shown which are needed regarding the investigation of the cadaver knee kinematics. The knee joint was resected with $10-15 \mathrm{~cm}$ parts of the femur and the tibia also. The joint is fixed to the apparatus with textile bakelite links (1) which are cemented into the medullary cavity. The link is cemented into the femur, while the femur itself is fixed to the base plate (2) of the apparatus, therefore the fixed femur is horizontally positioned. The loads acting on the joint are the weight load and the quadriceps muscle force in case of the studied motion type. The weight of the tibia was modeled with a concentrated force, which is connected to the tibia through a ball joint (3) screwed into the axis of the tibial link. The other force, the quadriceps 
muscle force was modeled by rubber-muscle model (4). The suitability of this was proven earlier.' This muscle model is connected to the body of the apparatus via a load cell (5) and a spindle (6) for setting the knee position, and the muscle tension. The movement of the tibia is also realized by the spindle. The change of the tibial position relative to the extended position of the knee was determined by the Polaris ${ }^{10}$ (Northern Digital Inc., Waterloo, Ontario, Canada) optical position measuring system after proper authentication. Two moving trackers were fixed to femur and the tibia. One of them was rigidly bolted to the fixed femur (7) and the other one was also rigidly bolted to the moving tibia. By this way they form a rigid body-system. The Polaris measuring system tracks the movement of the fixed trackers in the absolute coordinate system of the Polaris. During the experiment the tracker coordinate systems are rigidly fixed to the bones and they are continuously recorded by the Polaris. The position is described by six data: three coordinates of the origin in the absolute coordinate system and three angles which describes the position of the coordinate axes. These are the so-called Euler-angles.

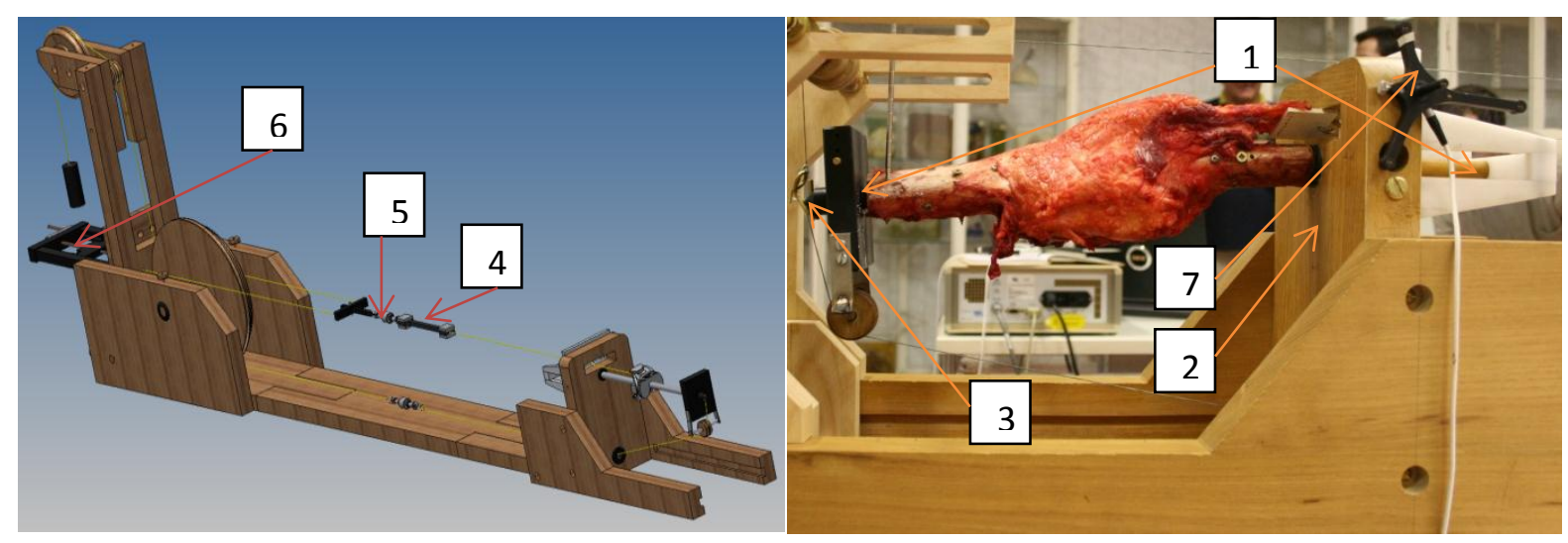

Figure 2. Cadaver knee test rig

\section{Method of experiments and evaluation}

In our experiments, the knee joints were obtained from five subjects between 28-60 years of age. Experiments were performed on seven knee joints in order to create the reference function (TUKEB 165-1/2002). The condition of the knee joints were controlled after the experiments. All the used knee joints were in suitable, undamaged condition.

Analysis was performed using anatomical coordinate system fixed to the bones for the comparison of the measurements on different subjects. However, there was a fundamental problem in the evaluation, due to the missing anatomical points of the resected knee joint. Therefore, the anatomical points had to be recorded (Figure 4) on the whole body before the resection of the knee joint. These points are easily palpable on the tibia of the whole body, so their detection can be solved by a simple pointer tracker. The determination of the center point of the femoral head is more complex. It can be determined by measuring the coordinates of the motion tracker fixed to the femur during the circling motion of the femur. A mention must be made that these points generally move on a sphere. The center of the sphere, which is the center of the femoral head (fh), can be calculated (Figure 4) from the coordinates of the origin of the tracker coordinate system. The other two necessary points on the femur are the epicondylar 
points. The described method was modified during the creation of the anatomical coordinate system. The centers of the femoral posterior condylar curves were used instead of the epicondylar points (Figure 3). The reason of this modification is also twofold. On the one hand, the epicondylar points are uninterpretable on prosthesis, and on the other hand, the accuracy of the epicondylar points' determination is much smaller than the accuracy of the determination of the posterior condylar curves' centers. ${ }^{11}$ Anatomical coordinate system defined by the VAKHUM project was fitted to the recorded anatomical points ${ }^{12}$ (Figure 4). Since the knee joint was fixed into the apparatus in resected state, the possibility of the transformation of resected anatomical points needed to be ensured. For this reason, the position of the secured markers, both in the femur and the tibia, were recorded. After these measurements the joint, which was fixed in the apparatus, was resected. The position of the anatomical points and the markers were recorded in the apparatus as well. In the following steps, continuous measurements were performed after the determination of the discrete points in order to determine the kinematics (flexion-rotation). The above-mentioned measurements were carried out in 3 repetitions per joint. These repetitions gave provided the same results, therefore the measurements are acceptably repeatable. ${ }^{13}$

The flexion-rotation data was determined by transformations using the transformation matrices. The matrices were generated from the positions of the anatomical points, the markers, and the current positions of the trackers. These transformation matrices can be generated from the data measured by the Polaris as well. These processes were carried out on basis of the Grood-Suntay's angle definition. ${ }^{14}$ The results can be drawn as the function of flexion.

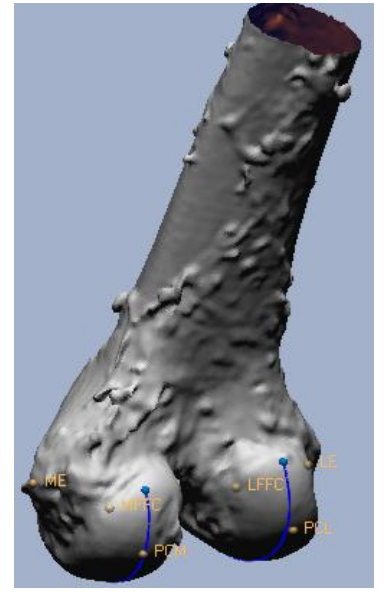

Figure 3. Anatomical points on the femur
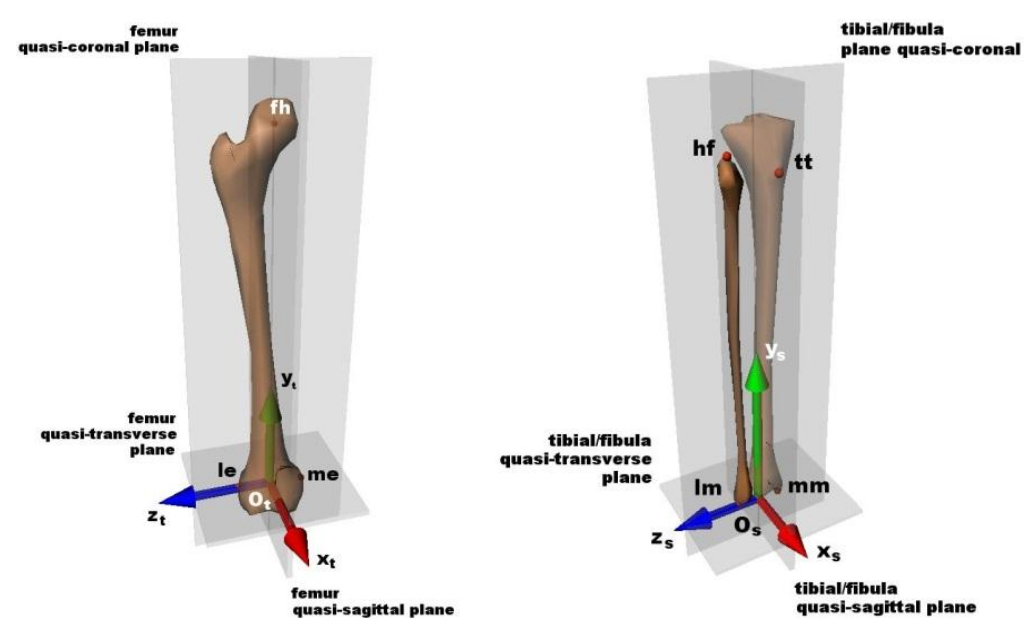

Figure 4. The anatomical coordinate system of the femur and the tibia

The literature suggests that during knee flexion the movement can be divided into two parts. The first $20-30^{\circ}$ section of the flexion is the section the screw-home motion, ${ }^{13}$ where the kinematics is governed mainly by the geometry. After this section the motion is continuously passing through to active functional $\operatorname{arc}^{13}$ where the movements of knee joint is controlled by the muscle forces collectively. Accordingly, tri-linear functions were fitted to the rotation-flexion data. The first section of motion extends between $0^{\circ}$ and $25^{\circ}$ of flexion angle. This is the section of screw-home motion. The boundary of the following two segments (at about $50^{\circ}$ of flexion angle) can be 
interpreted from Figure 5. The second section of the flexion extends between $25^{\circ}$ and $50^{\circ}$. This is a transitional section to unconstrained motion. The range over $50^{\circ}$ of flexion is the range of unconstrained motion, where the motion may be governed by the muscle forces.

The schematic diagram of the approximate functions can be seen on Figure 5. The fitting was based on the principle of least squares. The parameters of the fitted functions can be calculated from equation 1 to 9 .

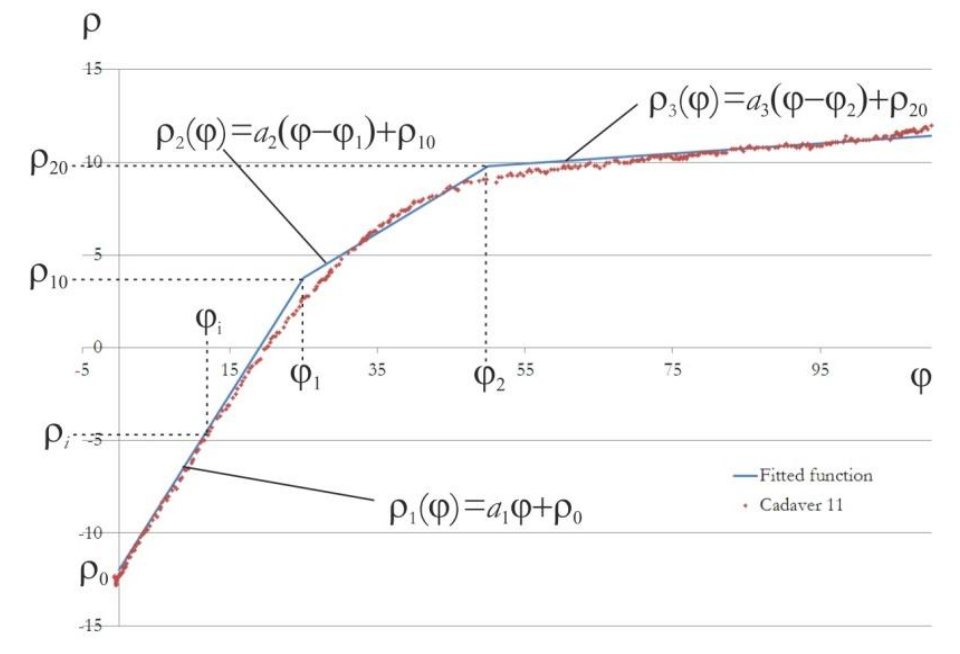

Figure 5. The trilinear function fitted to the measurement data on knee no. 7

First section: $0-25^{\circ}$ flexion

$$
\begin{gathered}
a_{1}=\frac{\sum_{i=1}^{n_{1}} \rho_{i} \cdot \varphi_{i}}{\sum_{i=1}^{n_{1}} \varphi_{i}^{2}} \\
\rho_{0}=\frac{\sum_{i=1}^{n_{1}} \rho_{i} \sum_{i=1}^{n_{1}} \varphi_{i}^{2}-\sum_{i=1}^{n_{1}} \varphi_{i} \sum_{i=1}^{n_{1}} \rho_{i} \varphi_{i}}{n_{1} \sum_{i=1}^{n_{1}} \varphi_{i}^{2}-\left(\sum_{i=1}^{n_{1}} \varphi_{i}\right)^{2}} \\
\rho_{1}(\varphi)=\rho_{0}+a_{1} \cdot \varphi
\end{gathered}
$$

Second section: $25-50^{\circ}$ flexion

$$
\begin{gathered}
a_{2}=\frac{\sum_{i=n_{1}+1}^{n_{2}} \rho_{i} \cdot\left(\varphi_{i}-\varphi_{1}\right)-\rho_{10} \sum_{i=n_{1}+1}^{n_{2}}\left(\varphi_{i}-\varphi_{1}\right)}{\sum_{i=n_{1}+1}^{n_{2}}\left(\varphi_{i}-\varphi_{1}\right)^{2}} \\
\rho_{10}=a_{1} \cdot \varphi_{1}+\rho_{0}
\end{gathered}
$$




$$
\rho_{2}(\varphi)=\rho_{10}+a_{2} \cdot\left(\varphi-\varphi_{1}\right)
$$

Third section: over $50^{\circ}$ flexion

$$
\begin{gathered}
a_{3}=\frac{\sum_{i=n_{2}+1}^{n_{3}} \rho_{i} \cdot\left(\varphi_{i}-\varphi_{2}\right)-\rho_{20} \sum_{i=n_{2}+1}^{n_{3}}\left(\varphi_{i}-\varphi_{2}\right)}{\sum_{i=n_{2}+1}^{n_{3}}\left(\varphi_{i}-\varphi_{2}\right)^{2}} \\
\rho_{20}=a_{2} \cdot\left(\varphi_{2}-\varphi_{1}\right)+\rho_{10} \\
\rho_{3}(\varphi)=\rho_{20}+a_{3} \cdot\left(\varphi-\varphi_{2}\right)
\end{gathered}
$$

where
$i$ : the sample of the measured data
$\varphi$ : flexion
$\rho$ : rotation

$a_{1} ; a_{2} ; a_{3}$ : the gradient of the fitting functions on the first, second and third sections

$\rho_{0}$ : the intersection of the fitting linear function along the first section

$\rho_{1}$ : the value of the rotation at the boundary of the first and second sections

$\rho_{2}$ : the value of the rotation at the boundary of the second and third sections

$\varphi_{1}$ : the value of the flexion at the boundary of the first and second sections $\left(\varphi_{1}=25^{\circ}\right)$

$\varphi_{2}$ : the value of the flexion at the boundary of the second and third sections $\left(\varphi_{2}=50^{\circ}\right)$

$n_{1} ; n_{2} ; n_{3}$ : the number of measured data on the first, second and third sections

Most of the authors treat the rotation as zero in the totally extended position (Figure 1). For the sake of the comparability, both the own measurement results and the fitting functions need to be transformed to zero.

If the points of the anatomical coordinate-system remain unchanged, then the degree of rotation is specified by the position of the malleolus points on the tibia. The two additional points which are needed for the tibial coordinate-system have virtually no effect on the curves, only the position of the longitudinal axis is determined by them. These points are close to each other and relatively far from the ankles, therefore the effect of changing in the range of the recording accuracy is minimal. If the positions of the malleolus points are changed then the curves shift parallel (Figure O). 


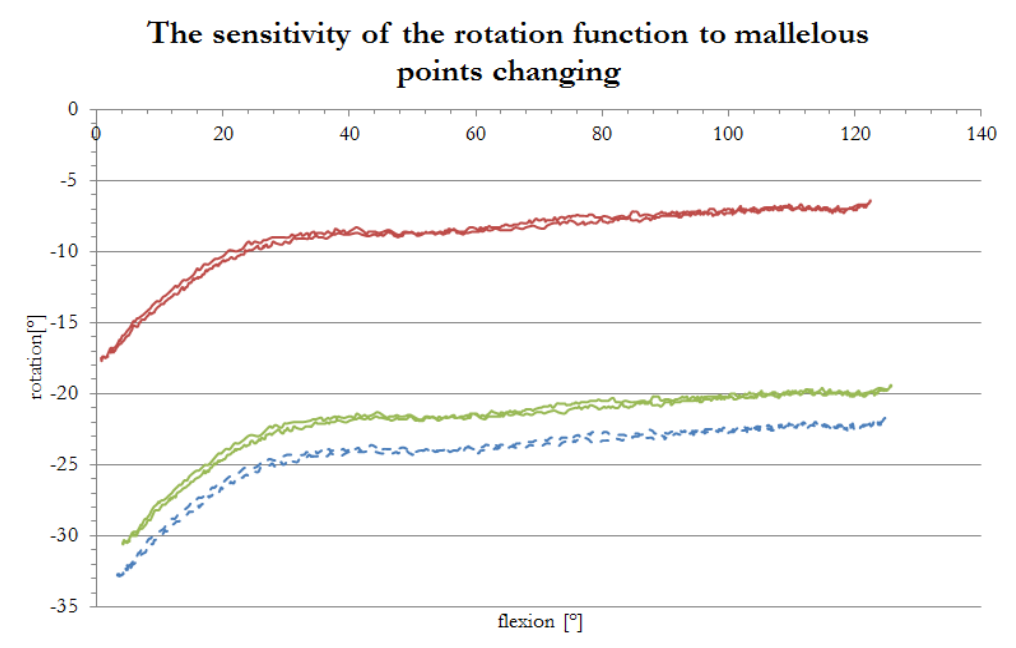

Figure 6. The sensitivity of the rotation function in case of knee no. 3

This phenomenon is interpreted as follows: according to the interpretation of Grood \& Suntay, the rotation is a so-called third angle, which describes how the coordinate-system of the tibia is rotated relative to the longitudinal axis of the femur.

Based on these above-mentioned reasons, the fitted rotation-flexion functions can be shifted parallel towards the rotation into the origin (Figure 7). If the measurement results begin from nonzero flexion, the rotation value of zero flexion is determined by the intersection of the fitted functions. With this method, the starting points of the measured rotation-flexion curves can be transformed to the origin.

\section{Results}

The functions fitted on experimental data (involving seven knee joints) are shown on Figure 7 after the transformation described above. These curves are drawn with thin line. The coefficients of the functions are included in Table 1. The reference function is obtained by averaging these functions, which is essential in the design of prostheses and in their qualification. The coefficients of the reference function are included in Table 2.

\begin{tabular}{|c|c|c|c|r|r|r|}
\hline$j$ & $a_{1 j}$ & $a_{2 j}$ & $a_{3 j}$ & $\rho_{0 j}\left[^{\circ}\right]$ & $\rho_{10 j}\left[^{\circ}\right]$ & $\rho_{20 j}\left[^{\circ}\right]$ \\
\hline 1 & 0,48 & 0,058 & 0,101 & $-24,2$ & $-12,1$ & $-10,6$ \\
\hline 2 & 0,42 & 0,094 & 0,048 & $-14,1$ & $-3,5$ & $-1,1$ \\
\hline 3 & 0,38 & 0,029 & 0,028 & $-18,3$ & $-8,7$ & $-7,9$ \\
\hline 4 & 0,47 & 0,123 & 0,047 & $-28,7$ & $-16,8$ & $-13,7$ \\
\hline 5 & 0,24 & 0,020 & $-0,032$ & $-13,4$ & $-7,4$ & $-6,8$ \\
\hline 6 & 0,61 & 0,088 & $-0,025$ & $-11,6$ & 3,6 & 5,9 \\
\hline 7 & 0,63 & 0,240 & 0,02723 & $-11,9$ & 3,7 & 9,7 \\
\hline
\end{tabular}

Table 1. The coefficients of the fitted functions 


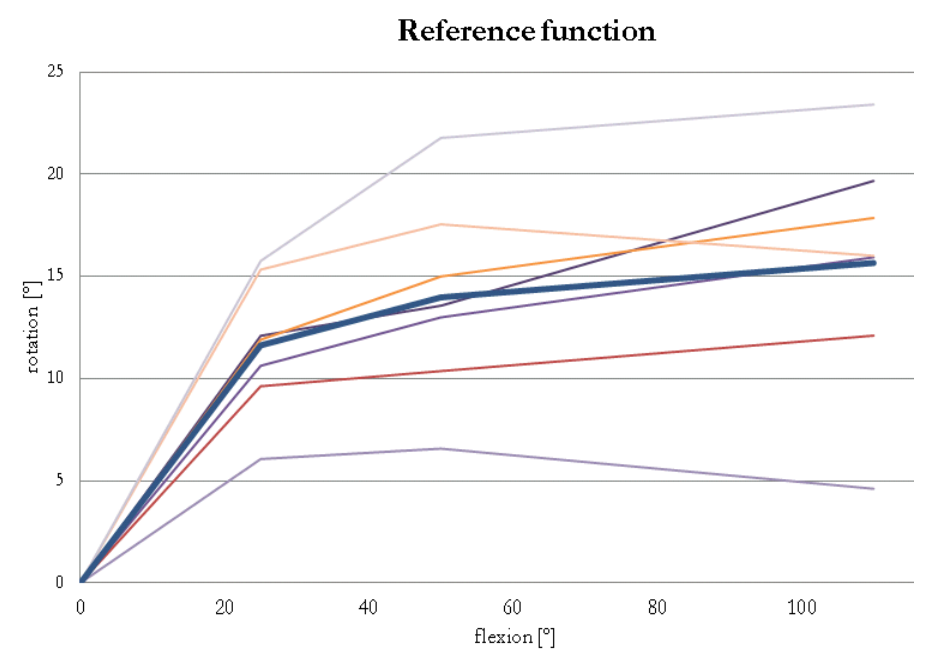

Figure 7. Reference function from the seven fitted and transformed linear functions

\begin{tabular}{|c|c|c|c|c|c|c|}
\hline & $a_{1}$ & $a_{2}$ & $a_{3}$ & $\rho_{0}\left[^{\circ}\right]$ & $\rho_{10}\left[^{\circ}\right]$ & $\rho_{20}\left[^{\circ}\right]$ \\
\hline Reference function & $\mathbf{0 , 4 6}$ & $\mathbf{0 , 0 9 3}$ & $\mathbf{0 , 0 2 8}$ & $\mathbf{0}$ & $\mathbf{1 1 , 6}$ & $\mathbf{1 3 , 9}$ \\
\hline
\end{tabular}

Table 2. The coefficients of the reference function

\section{Discussion, Conclusion}

Overall as new results we can present:

- an earlier designed experimental apparatus,

- a protocol for determining the anatomical coordinate system,

- determination of a tri-linear rotation-flexion function which approximates the measured data and can be specified in segments,

- a function based on a large number of experiments, which describes the change of rotation on an average human knee joint.

The results can be utilized in prosthetic design in a way, that the rotation carried out by the prosthesis should approximate this function.

\section{REFERENCES}

1. Fekete G, De Baets P, Wahab MA, Csizmadia BM, Katona G, Vanegas-Useche LV, Solanilla JA. Sliding-rolling ratio during deep squat with regard to different knee prostheses. Acta Polytechnica Hungarica 2012;9(5):5-24.

2. Baldwin MA, Clary C, Maletsky LP, Rullkoetter PJ. Verification of predicted specimen-specific natural and implanted patellofemoral kinematics during simulated deep knee bend. Journal of Biomechanics 2009;42(14):2341-8.

3. Wilson DR, Feikes JD, Zavatsky AB, O'Connor JJ. The components of passive knee movement are coupled to flexion angle. Journal of Biomechanics 2000;33:465-73. 
4. Bull AMJ, Kessler O, Alam M, Amis AA. Changes in Knee Kinematics Reflect the Articular Geometry after Arthroplast. Clinical Orthopaedics and Related Research 2008;466:2491-9.

5. Most E, Axe J, Rubash H, Li G. Sensitivity of the knee joint kinematics calculation to selection of flexion axes. Journal of Biomechanics 2004;37:1743-8.

6. Akalan NE, Ozkan M, Temelli Y. Three-dimensional knee model: Constrained by isometric ligament bundles and experimentally obtained tibio-femoral contacts. Journal of Biomechanics 2008;41:890-6.

7. Wilson DR, Feikes JD, O'Connor JJ. Ligaments and articular contact guide passive knee flexion. Journal of Biomechanics 1998;31:1127-36.

8. Tung-Wu L, Tsung-Yuan T, Mei-Ying K, Horng-Chaung H, Hao-Ling C. In vivo three-dimensional kinematics of the normal knee during active extension under unloaded and loaded conditions using single-plane fluoroscopy. Medical Engineering \& Physics 2008;30:1004-12.

9. Csizmadia B, Katona G. Some result of the motion analysis executed on experimental model of the knee. In: A. Tőróková editor. Proceedings of 23rd Danubia-Adria Symposium on Experimental Methods in Solid Mechanics; 2006. Sept. 26-29; Zilina, Slovak Republic. Zilina: EDIS, 2006. p. 145-6.

10.NDI, http://www.ndigital.com/medical/polarisfamily-techspecs.php

11. Donald Eckhoff MD, Craig Hogan MD, Laura DiMatteo MD, Mitch Robinson MD, Bach J. Difference Between the Epicondylar and Cylindrical Axis of the Knee. Clinical Orthopaedics and Related Research 2007,461:238-44.

12. Hilal I, Van Sint Jan S, Leardini A, Della Croce U. D3.2. Technical Report on Data Collection Procedure ANNEX I. 20. p. In: Virtual Animation of the Kinematics of the Human for Industrial, Educational and Research Purposes. Information Societies Technology Programme. 2002.

13. Katona G, Csizmadia BM, Bíró I, Andrónyi K, Krakovits G. Motion analysis of human cadaver kneejoints using anatomical coordinate system. Biomechanica Hungarica 2010;3(1):93-100.

14. Grood ES, Suntay WJ. A joint coordinate system for the clinical description of 3-dimensional motions - application to the knee. Journal of Biomechanical Engineering-Transactions of the Asme 1983; 105(2):136-44.

15. Freeman MAR. How the knee moves. Current Orthopaedics 2001;15(6):444-50.

The research was supported by the TÁMOP-4.2.2.B-10/1-2010-0011 „Development of a complex educational assistance/support system for talented students and prospective researchers at the Szent István University" project. 